Check for updates

Cite this: RSC Adv., 2017, 7, 24988

Received 7th March 2017 Accepted 24th April 2017 DOI: $10.1039 / \mathrm{c} 7 \mathrm{ra0} 2774 \mathrm{e}$

rsc.li/rsc-advances

\title{
Quantitative analysis of yeast growth process based on FT-NIR spectroscopy integrated with Gaussian mixture regression
}

\author{
Wei Wang, ${ }^{a}$ Hui Jiang, (DD *a Guohai Liu, ${ }^{\text {a }}$ Quansheng Chen, ${ }^{\mathrm{b}}$ Congli Mei, ${ }^{\mathrm{a}}$ Kangji Li ${ }^{\mathrm{a}}$ \\ and Yonghong Huang ${ }^{a}$
}

To improve the yield of industrial fermentation, herein, we report a method based on Fourier-transform near-infrared spectroscopy (FT-NIR) to predict the growth of yeast. First, the spectra were obtained using an FT-NIR spectrometer during the process of yeast cultivation. Each spectrum was acquired over the range from 10000 to $4000 \mathrm{~cm}^{-1}$, which resulted in spectra with 1557 variables. Moreover, the optical density (OD) value of each fermentation sample was determined via photoelectric turbidity method. Then, using a method based on competitive adaptive reweighted sampling (CARS), characteristic wavelength variables were selected from the preprocessed spectral data. Gaussian mixture regression (GMR) algorithm was employed to develop the prediction model for the determination of OD. The results of the model based on GMR were achieved as follows: only 13 characteristic wavelength variables were selected by CARS, the coefficient of determination $R_{\mathrm{p}}{ }^{2}$ was 0.98842 , and the root mean square error of prediction (RMSEP) was 0.07262 in the validation set. Finally, compared to kernel partial least squares regression (KPLS), support vector machine (SVM), and extreme learning machine (ELM) models, GMR model showed excellent performance for prediction and generalization. This study demonstrated that FT-NIR spectroscopy analysis technology integrated with appropriate chemometric approaches could be utilized to monitor the growth process of yeast, and GMR revealed its superiority in model calibration.

\section{Introduction}

Owing to the shortage of fossil energy sources in the world, development of biomass energy has gained significant attention. Yeast fermentation, which is one of the most common ways of biomass energy production, has been widely applied in the alcohol industry. ${ }^{1-3}$ In the industrial production process, prediction of growth process of yeast can not only help people know the period of yeast growth, but can also be used to more accurately select the termination time of yeast culture to maximize the production and compare cell growth status of different batches to choose the best feeding time. Therefore, it plays a significant guiding role in the fermentation industry. At present, some main methods such as cell count technique, plate colony-counting, and weighing have been reported to predict the growth process of yeast cells. However, detection process via these methods is tedious and these methods require usage of certain chemical reagents that will lead to the destruction of the

${ }^{a}$ School of Electrical and Information Engineering, Jiangsu University, Zhenjiang 212013, PR China. E-mail: h.v.jiang@ujs.edu.cn; h.v.jiang@hotmail.com; Fax: +86 511 88780088; Tel: +86 51188791245

${ }^{b}$ School of Food and Biological Engineering, Jiangsu University, Zhenjiang 212013, PR China samples, environmental pollution, and waste of time. ${ }^{4}$ Therefore, a rapid and reliable analytical method is essentially required to predict the growth process of yeast to assure the quality and consistency of the product of yeast.

Fourier-transform near infrared (FT-NIR) spectroscopy can possibly serve as a noninvasive technique for the quantitative analysis of the growth process of yeast as it interacts with molecular groups associated with process parameters such as biomass ( $\mathrm{C}-\mathrm{H}$ group), organic acid and moisture ( $\mathrm{O}-\mathrm{H}$ group), and scattering from microstructures., ${ }^{5,6}$ Most of the nearinfrared absorption bands associated with these groups are overtone or combination bands of the fundamental absorption bands in the mid-infrared regions, which are due to vibrational and rotational transition. ${ }^{7}$ In recent years, FT-NIR spectroscopy technology has been applied in the field of yeast fermentation. ${ }^{8,9}$ The abovementioned studies show that FT-NIR spectroscopy is a highly potential technique for the analysis of the growth process of yeast.

However, FT-NIR spectroscopy analysis technique is an indirect measurement technique. In recent years, a number of studies have shown that near infrared spectral information has complicated backgrounds with peak overlapping and weak signal. Generally, NIR has hundreds of variables, and some uninformative variables, redundant variables, and serious 
multicollinearity exist among the wavelength variables. Model calibration using complete spectral data will not only reduce the modeling speed, but also affect the accuracy and robustness of the model. Therefore, it is necessary to screen the spectral characteristic wavelength variables by an appropriate wavelength variable selection method prior to model calibration. ${ }^{10}$

Additionally, the application of a proper multivariate analysis method in model calibration has been proven to be greatly beneficial for providing more reliable and parsimonious model. During the last few decades, many different algorithms, such as partial least squares regression (PLS), ${ }^{11}$ kernel partial least squares regression (KPLS), ${ }^{12}$ neural network (NN), ${ }^{13}$ support vector machine (SVM) ${ }^{14}$ extreme learning machine (ELM) ${ }^{15}$ mixture Poisson regression (MPR), ${ }^{16}$ and Gaussian mixture regression $(\mathrm{GMR})^{17}$ have been developed for model calibration. Among these, GMR is a relatively new algorithm, which not only has the advantages of smaller calculation quantity and few parameters, but also is suitable for dealing with the problem of non-normal distribution. ${ }^{17}$ Thus, in this study, GMR was applied to construct a regression model for the prediction of the growth process of yeast.

In the process of microbial culture, optical density (OD) is often used as an index to reflect the growth state of a microorganism. ${ }^{18}$ Therefore, in this study, FT-NIR spectroscopy technique combined with proper multivariate data analysis was employed to carry out quantitative analysis on the growth process of yeast culture (i.e. OD values). The specific objectives of this study were

(1) to eliminate suspended particles, surface astigmatism, and optical path change by SNV;

(2) to filter out the characteristic information variables and compressed spectral data dimension by CARS;

(3) to use optimal spectral data for the construction of a prediction model via Gaussian mixture regression (GMR).

To highlight the superiority of the prediction precision of GMR algorithm adopted in this study, the results of the GMR model were compared with those of other three different regression algorithms: kernel partial least squares, KPLS; support vector machine, SVM; and extreme learning machine, ELM. Simultaneously, the parameters of the models were optimized via a cross-validation method.

\section{Materials and methods}

\subsection{Yeast cultivation and sample division}

After culture expansion of yeast, sterile malt medium and yeast suspension were transferred into volumetric flasks. First, three $250 \mathrm{ml}$ volumetric flasks were marked as I, II, and III, and then, $125 \mathrm{ml}$ malt extract medium and $0.5 \mathrm{ml}$ yeast suspension were loaded into each of the volumetric flasks. Finally, yeast in these three volumetric flasks was continuously cultured for 72 hours in a constant temperature shock incubator, and the temperature and rotation rate of the incubator was set at $28{ }^{\circ} \mathrm{C}$ and $110 \mathrm{rpm}$, respectively. Based on the abovementioned experimental steps, 5 sets of yeast culture experiments were carried out. In this way, we could obtain 6 sets of experimental data.
For each set of yeast culture experiment, sampling was carried out at 19 different time points during the yeast culture, from loading to the end of culture $(0,4,8,12 \ldots 72 \mathrm{~h})$. In addition, to avoid contamination of sterile malt medium by multiple sampling, 19 sampling time points were divided into three parts: the first 7 sampling time points $(0,4,8,12,16,20$, and $24 \mathrm{~h})$ were executed in the volumetric flasks numbered as I, the next 6 sampling time points $(28,32,36,40,44$, and $48 \mathrm{~h})$ were implemented in the volumetric flasks numbered as II, and the last 6 sampling time points $(52,56,60,64,68$, and $72 \mathrm{~h})$ were carried out in the volumetric flasks numbered as III. Thus, 19 samples were obtained for each set of experiment, and data from a total of 114 samples were obtained in 6 groups. Moreover, these four sets of experimental data were chosen as the training set, and the remaining two sets were used as the validation set.

\subsection{Measurement of the OD value}

First, the wavelength of the spectrophotometer was set at $600 \mathrm{~nm}$ and the light transmittance was adjusted to $100 \%$. Then, $1 \mathrm{~cm}$ cuvette was charged with $3.5 \mathrm{ml}$ of sterile malt extract medium as a control group. Since yeast culture is a dynamic process, to avoid the effect of yeast on the fermentation broth, 114 fermented samples were filtered using 0.45 microliter of microporous membrane. At each sampling point, $1 \mathrm{~cm}$ cuvette charged with $3.5 \mathrm{ml}$ of sterile fermented sample was used to measure the OD value by the spectrophotometer. Each sample was measured three times, and the three OD values were averaged to obtain a mean value. During the measurement, if the bacterial suspension is too thick, it should be appropriately diluted, such that the OD value remains between 0.1 and 0.65 . Table 1 shows the distribution of 114 samples in the training and validation sets.

\subsection{FT-NIR spectra acquisition and preprocessing}

FT-NIR spectral data were obtained in the transmittance mode using an Antaris ${ }^{\mathrm{TM}}$ II Fourier-transform near infrared (FT-NIR) spectrophotometer (Thermo Electron Co., USA). Each spectrum is an ensemble average of 32 scans in a quartz cuvette (Perkin Elmer., USA) with a $6 \mathrm{~mm}$ optical path. The spectral data were acquired in the range from 10000 to $4000 \mathrm{~cm}^{-1}$, which fetched the spectra with 1557 variables (resolution: $8 \mathrm{~cm}^{-1}$ ). To obtain more accurate spectral data, three different positions of each sample were acquired, and then, mean of these three spectral data was obtained as the raw spectral data for a sample. This was employed to construct the analysis model. Since the spectrometer was sensitive to changes in the environmental

Table 1 Descriptive statistics of OD in the training and validation sets

\begin{tabular}{lcccll}
\hline \multicolumn{5}{c}{ OD value } \\
\cline { 3 - 6 } Subsets & S.N. $^{a}$ & Mean & Maximum & Minimum & S.D. ${ }^{b}$ \\
\hline Training set & 76 & 1.3967 & 2.15 & 0.1230 & 0.7796 \\
Validation set & 38 & 1.4082 & 2.15 & 0.1250 & 0.7919 \\
${ }^{a}$ S.N.: sample number. ${ }^{b}$ S.D.: standard deviation. &
\end{tabular}


conditions such as temperature and humidity, the temperature was maintained around $25{ }^{\circ} \mathrm{C}$ at a steady humidity level in the laboratory.

Fig. 1(a) shows the raw FT-NIR spectra of the 114 yeast cultivation samples. FT-NIR spectra are affected by multifarious conditions such as changes in temperature, diffusion of light, a baseline shift or instrument noise. ${ }^{8}$ In addition, FT-NIR spectra contain chemical as well as physical information, which can be useless or mask important information. ${ }^{19}$ Therefore, to ensure the predicted effect of the calibration model, it was essential to select a suitable pretreatment method to weaken the physical and chemical interference. At present, many spectral preprocessing methods such as first and second derivative, standard normal variate transformation (SNV), and multiplicative scatter correction (MSC) have been reported. On comparing these spectral preprocessing, SNV was found to be superior to others in this study. In this experiment, a gap or bubble among yeast culture media was observed in the cuvette, which resulted in scattering of light. SNV has advantages with respect to correcting scattered light and removal of slope variation. Therefore, SNV was employed for light scatter correction and reducing the changes of light path length in the proposed work. SNV preprocessing spectra is presented in Fig. 1(b).
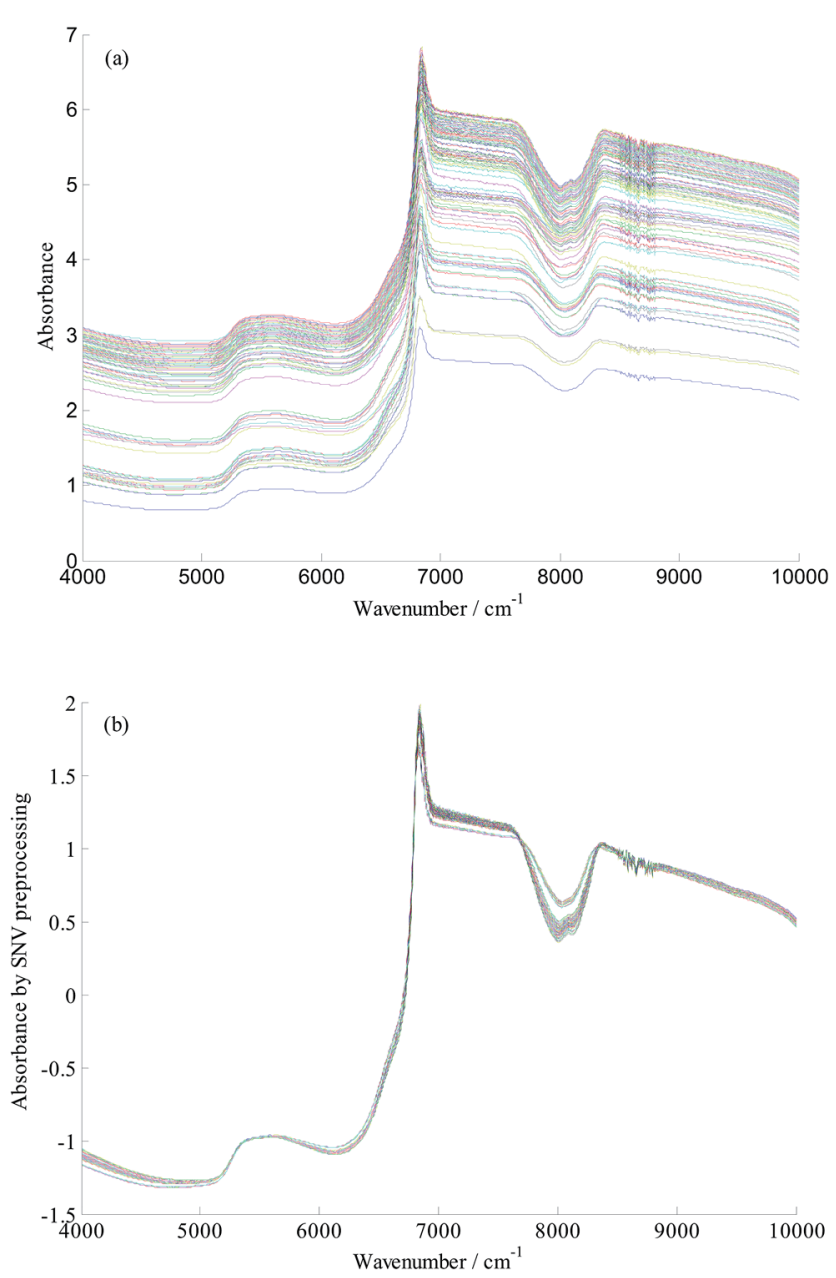

Fig. 1 Raw spectra (a) and SNV preprocessing spectra (b) of all the samples.

\subsection{Multivariate data analysis}

2.4.1 Wavelength variable selection. Modern chemometric studies have indicated that appropriate variable selection methods are essential for multivariate data analysis. ${ }^{20}$ The competitive adaptive reweighted sampling (CRAS) algorithm is a newly developed strategy for wavelength selection, employing the simple but effective principle survival of the fittest on which Darwin's evolution theory is based. ${ }^{21}$ Studies have shown that CARS is very effective in the selection of spectral data. ${ }^{22}$ Each wavelength variable was found to compete with others by its own weight, and some wavelength variables whose weights were relatively small were removed.

CARS can work in four successive steps: ${ }^{23}$

Step 1. Monte Carlo approach was applied for model sampling, $80 \%$ of the sample were randomly selected to build the PLS model, and the regression coefficient $\beta$ of the corresponding model was retained. The weight $w_{i}$ of the $i^{\text {th }}$ variable can be defined as follows:

$$
w_{i}=\frac{\left|\beta_{i}\right|}{\sum_{i=1}^{P}\left|\beta_{i}\right|}, i=1,2 \ldots, P
$$

Step 2. Exponentially decreasing function was employed to perform enforced wavelength selection. Wavelength retention rate was directly calculated using the following algorithm:

$$
r_{i}=a \mathrm{e}^{-k i}
$$

where $a=(p / 2)^{1 /(N-1)}, k=[\ln (p / 2)] /(N-1)$, and $P$ is the raw wavelength variables.

Step 3. The adaptive reweighted sampling (ARS) method was adopted to realize a competitive selection of wavelengths. Wavelength variables of the larger weights were selected to form subsets of wavelengths. After repeating this step for $\mathrm{N}$ times, CARS sequentially selected $\mathrm{N}$ subsets of wavelengths to build the PLS model.

Step 4. 5-Fold cross validation method was utilized to evaluate the subset. The subset with lowest RMSECV value was chosen as the optimal subset.

2.4.2 Gaussian mixture regression (GMR). In this section, the derivation of Gaussian mixture regression (GMR) was simply introduced, and further details regarding the GMR algorithm can be found in the literature. ${ }^{\mathbf{2 4 - 2 6}}$ The derivation of GMR was mainly based on the Gaussian conditioning and linear combination properties of Gaussian distributions. We speculated that $X$ indicated the space of the explanatory variables and $Y$ indicated the space of the response variables. $x$ is the input of the training data $(x \in X)$ and $y$ is the ideal output data $(y \in Y)$. For the given values of $x$ and $y$, the joint probability density can be formulated as follows:

$$
f_{x y}(x, y)=\sum_{j=1}^{K} \pi_{j} \phi\left(x, y ; \quad \mu_{j}, \sum_{j}\right)
$$


In addition, mean and covariance can be divided into input and output parts as follows:

$$
\mu_{j}=\left[\begin{array}{l}
\mu_{j x} \\
\mu_{j y}
\end{array}\right], \quad \sum_{j}=\left[\begin{array}{ll}
\sum_{j X X} & \sum_{j X Y} \\
\sum_{j X X} & \sum_{j Y Y}
\end{array}\right]
$$

where $\varphi\left(x, y ; \mu_{j}, \Sigma_{j}\right)$ denotes the probability density function of the multivariate GMM. Eqn (3) includes all the parameters such as the number of the mixture components $K$, prior $\pi_{j}$, mean value $\mu_{j}$, and variance of each Gaussian component $\Sigma_{j}$, which have been indicated as $\theta=\left(\theta_{1}, \theta_{2}, \ldots, \theta_{k}\right)$ with $\theta_{j}=\left(\pi_{j}, \mu_{j}, \Sigma_{j}\right)$ and the constraint $\sum_{j=1}^{K} \pi_{j}=1\left(0 \leq \pi_{j} \leq 1\right)$.

The marginal probability density $f_{X}(x)$ and mixing weight $w_{j}(x)$ can be calculated by ${ }^{27}$

$$
\begin{gathered}
f_{X}(x)=\int f_{X, Y}(x, y) \mathrm{d} y=\sum_{j=1}^{K} \pi_{j} \phi\left(x ; \quad \mu_{i X}, \quad \sum_{j X}\right) \\
w_{j}(x)=\frac{\pi_{j} \phi\left(x ; \quad \mu_{j X}, \quad \sum_{j X}\right)}{\sum_{j=1}^{K} \pi_{j} \phi\left(x ; \quad \mu_{j X}, \quad \sum_{j X}\right)}
\end{gathered}
$$

From eqn (3)-(5), we can obtain the global GMR function as

$$
f_{Y / X}(y / x)=\frac{f_{X Y(x, y)}}{f_{X}(x)}=\sum_{j=1}^{K} w_{j}(x) \phi\left(y ; m_{j}(x), \sigma_{j}{ }^{2}\right)
$$

The mean and variance of the conditional distribution can be estimated as follows:

$$
\begin{gathered}
m_{j}(x)=\mu_{j X}+\sum_{j Y X} \sum_{j X}^{-1}\left(x-\mu_{j X}\right) \\
\sigma_{j}^{2}=\sum_{j Y Y}-\sum_{j Y X} \sum_{j X}^{-1} \sum_{j X Y}
\end{gathered}
$$

For a given input variable, its prediction can be achieved by calculating the expectation over the conditional distribution $f_{Y / X}(y / x)^{27}$

$$
E\left[f_{Y / X}(y / x)\right]=\sum_{j=1}^{K} w_{j}(x) m_{j}(x)
$$

To build a GMM, the mixture components $K$ were set as 4 and the unknown parameter set $\theta$ of probabilistic weights were estimated first. Therefore, the maximum likelihood estimation (MLE) and expectation-maximization (EM) algorithm were adopted to optimize the parameters. With a set of given data, $(X$, $Y$ ) can be realized by estimating the model parameters $\theta$ in eqn (3). By maximizing the log-likelihood function $L\left(\theta_{k}\right)$, this process can be can realized, and the calculation formula can be expressed as $^{28}$

$$
L\left(\theta_{k}\right)=\ln \prod_{i=1}^{N} p\left(x_{i}, y_{i}\right)=\sum_{i=1}^{N} \ln \sum_{j=1}^{K} \pi_{j} \phi\left(x, y ; \mu_{j}, \sum_{j}\right)
$$

For the given training data, $\theta$ was calculated by maximizing this function via the ELM algorithm in the iterative means. It included two steps: ${ }^{29}$

(1) E step (expectation step):

$$
\begin{aligned}
p^{(s)}\left(l_{k} / x_{i}\right)= & \frac{\pi_{j}^{(s)} \phi\left(X, \mu_{j}^{(s)}, \sum_{j}^{(s)}\right)}{p^{(s)}\left(x_{i}, \theta\right)} \\
& (i=1,2, \ldots N ; j=1,2, \ldots k)
\end{aligned}
$$

where $p^{(s)}\left(l_{k} / x_{i}\right)$ denotes the posterior probability of the $i^{\text {th }}$ training sample within the $k^{\text {th }}$ Gaussian component at the $s^{\text {th }}$ iteration.

(2) M step (maximum step):

$$
\begin{gathered}
\mu_{j}^{(s+1)}=\frac{1}{\pi_{j} N} \sum_{i=1}^{N} p^{(s)}\left(l_{k} / x_{i}\right) x_{i} \\
\sum_{j}^{(s+1)}=\frac{1}{\pi_{j} N} \sum_{i=1}^{N} p^{(s)}\left(l_{k} / x_{i}\right)\left[\left(x_{i}-m_{j}\right)\left(x_{i}-m_{j}\right)^{T}\right] \\
\pi_{j}^{(s+1)}=\frac{1}{N} \sum_{i=1}^{N} p^{(s)}\left(l_{k} / x_{i}\right)
\end{gathered}
$$

where $\mu_{j}^{(s+1)}, \Sigma_{j}^{(s+1)}$, and $\pi_{j}^{(s+1)}$ are the mean, covariance, and prior probability of the $k^{\text {th }}$ Gaussian component at the $(s+1)^{\text {th }}$ iteration, respectively.

\subsection{Software}

All the algorithms were implemented in Matlab R2012a (Mathworks, Natick, USA) on Windows 7. The GMR matlab codes were downloaded from http://www.pudn.com/ for free of charge.

\section{Results and discussion}

\subsection{Efficient variable selection by CARS}

Before model calibration, efficient variables were selected first by CARS algorithm for a simplified model and improving the precision. In the calculations of CARS, the number of Monte Carlo sampling runs was 50, the maximum number of latent variables to be extracted, and the group number for the $K$-fold cross validation were all set at 5 . The data processing method was selected as center.

Fig. 2(a) shows the process of the characteristic wavelength selection by CARS. It can be seen from the graph of the relationship between the number of reserved wavelengths and the number of sampling runs that with the increase in the number of runs, the selected wavelength variables present a decreasing trend. This trend was initially rapid and then slowed down, thereby reflecting the process of rough and careful selection of variables. Fig. 2(b) shows the variation trend chart of the root mean square error of cross validation (RMSECV), wherein, it can be seen that RMSECV first descends and then ascends. When 

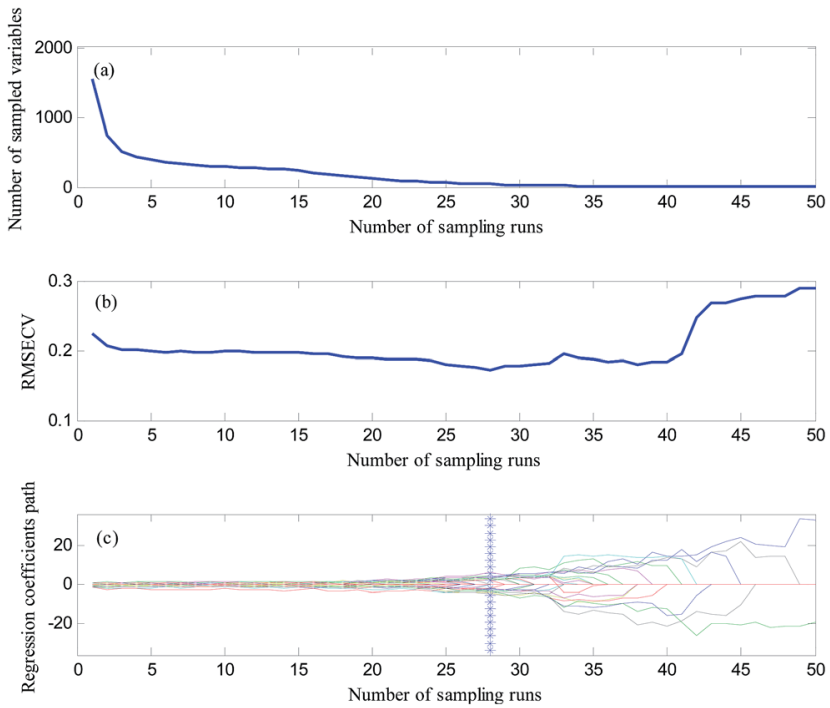

Fig. 2 (a) The changes of the number of selected wavelength variables, (b) the changes of RMSECV and (c) the regression coefficients of each wavelength variables during the calculations of CARS algorithm.

the number of sampling runs was 28, RMSECV attained the minimum value at 0.1736 . After $28^{\text {th }}$ time sampling, some of the relevant variables started to disappear, thereby increasing the RMSECV value. In Fig. 2(c), “*” perpendicular to the horizontal axis indicates that the minimum value of RMSECV was obtained on $28^{\text {th }}$ time sampling. According to the principle of minimum RMSECV, 13 characteristic variables were selected at last. Fig. 3 shows the distribution of the 13 selected characteristic variables in the entire spectral region after the CARS operation.

\subsection{GMR modeling and prediction results}

The GMR algorithm was employed to build a validation mode using the 13 selected characteristic variables by CARS for quantitative analysis. The capabilities of each GMR model were evaluated according to the coefficients of determination $\left(R_{\mathrm{p}}{ }^{2}\right)$ and the root mean square error of prediction (RMSEP) in the validation set. GMR model was developed using the selected

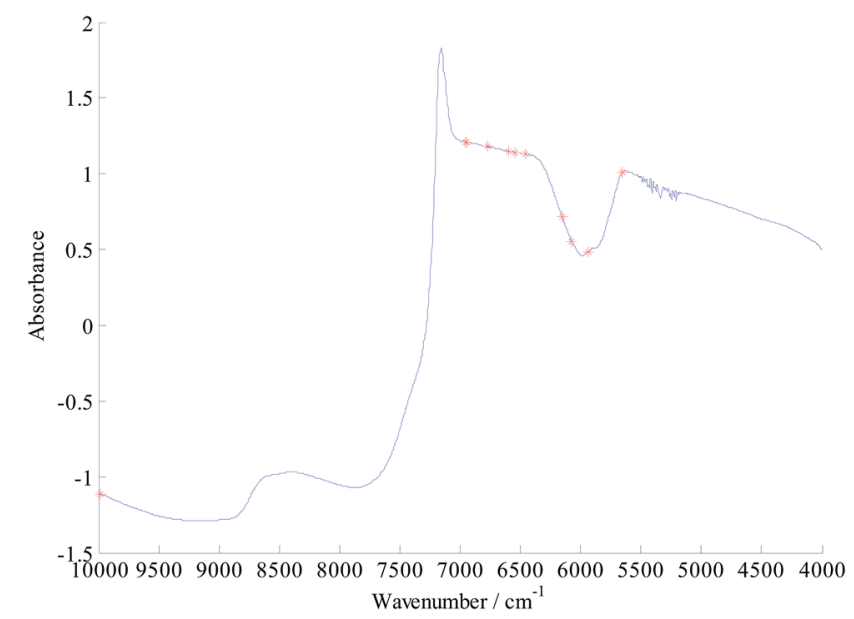

Fig. 3 Distribution of variables (shown by ' ${ }^{\prime}$ ) chosen by the CARS method.

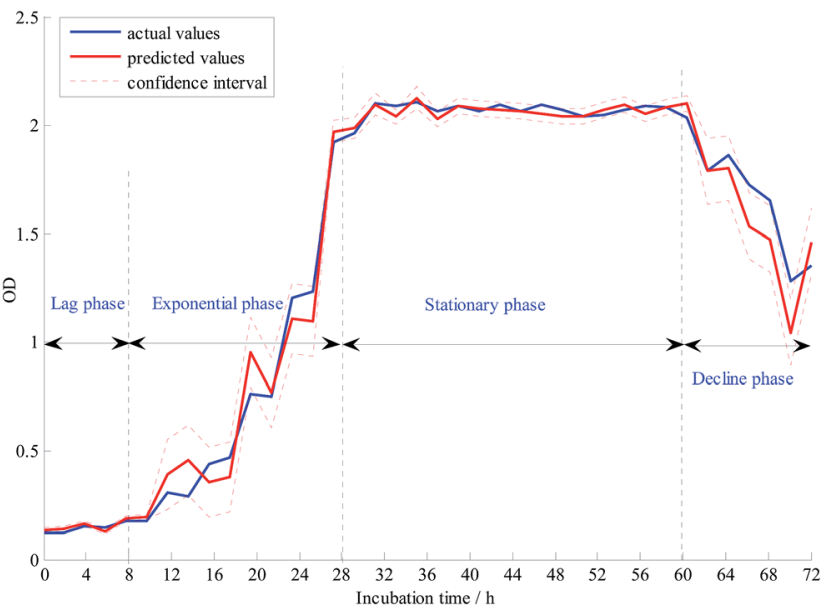

Fig. 4 Prediction of the yeast growth process using the GMR model.

characteristic variables by CARS algorithm. Fig. 4 shows the prediction effect of GMR model, the prediction is expressed as mean with $2 *$ std (std, standard deviation) error bars (red dotted lines). The regions between the two red dotted lines depict the confidence intervals. In Fig. 4, the red line was found to be very close to the blue line, and the confidence intervals were very small. All these phenomena demonstrated the modeling ability of GMR. The value of RMSEP of GMR model was 0.07262 and $R_{\mathrm{p}}^{2}$ was 0.98842 in the validation set.

\subsection{Comparison of different models}

To show that GMR has a better predictive performance, it was compared with KPLS, SVR, and ELM approaches in the present study. Table 2 shows the best predicted results obtained via KPLS, SVR, ELM, and GMR approaches in the validation set. As shown in Table 2, the RMSEP of GMR and mean of confidence interval (M.C.I) were less than those of other models, and $R_{\mathrm{p}}{ }^{2}$ was found to be higher than that of other models. These results implied that the GMR algorithm has a good generalization performance in model calibration, and another advantage of GMR is that it not only provides accurate prediction results, but also the smallest mean of confidence interval of the results obtained.

In addition, through the comparison of these methods, we found that there are several explanations for this phenomenon. KPLS and SVR are the common techniques for the regression of complex non-linear data sets. The key to this model is to map

Table 2 Results and comparison of the KPS, SVR, ELM, and GMR models in the validation set

\begin{tabular}{llll}
\hline Models & RMSEP & $R_{\mathrm{p}}{ }^{2}$ & M.C.I. $^{a}$ \\
\hline KPLS & 0.47808 & 0.91521 & 0.6001 \\
SVR & 0.19721 & 0.93804 & 0.3658 \\
ELM & 0.22983 & 0.92088 & 0.4502 \\
GMR & 0.07262 & 0.98842 & 0.1560
\end{tabular}

${ }^{a}$ M.C.I.: mean of confidence interval. 
the data in a higher dimensional feature space using kernel transformation. However, the disadvantage of using this kernel function is that the correlation between the obtained regression model and the original input space is lost. As a result, it may lose some useful information variables, which would cause decline in the prediction precision of the model. Moreover, because of the application of the kernel function, the running time KPLS and SVR program is longer than that of other models. ELM as compared to traditional neural network methods has simple structure, high learning speed, and good generalization performance; however, the dimension of the spectral data is usually very high while more hidden nodes should be incorporated in the original ELM model for spectral data. Therefore, the output matrix of the hidden layer of ELM model appeared as a high dimensional and high collinearity problem due to yeast growth in a complex environment. The process data did not originate from a single operating region; moreover, data distribution may be complicated with arbitrary non-Gaussian patterns. As a mixture model can represent arbitrarily complex probability density functions, GMR is one of the ideal tools for modeling complex multi-class dataset. Moreover, GMR not only has the tight structure of a parametric model, but also still retains the flexibility of a nonparametric model. Considering sufficient linear combinations of basis single multivariate Gaussian distribution, GMM can smoothen the probability distribution of arbitrary shape. Therefore, GMR reflected excellent generalization in its theory, which brings a slightly better prediction effect than the other regression algorithms.

\section{Conclusions}

In this study, a Gaussian mixture regression model based on FTNIR spectroscopy technique was constructed for quality prediction in the growth progress of yeast. To improve the GMR model fitting, SNV was first used to preprocess the spectra data, and then, the characteristic variables were extracted by the CARS approach. Compared to other conventionally used quantitative analysis approaches (KPLS, SVR, and ELM), GMR exhibited faster computation speed and higher generalization performance.

This study not only broadens the scope of CARS and GMR algorithm's application, but also provides a new theoretical basis for the rapid and non-destructive detection of microbial growth process. Moreover, it also makes a reference in the research on the improvement of the fermentation technology informationization and intelligent monitoring of other fermentation processes and has broad application prospect.

\section{Acknowledgements}

The authors gratefully acknowledge the financial support provided by the Natural Science Foundation of Jiangsu Province (Grant No. BK20140538, BK20130531, BK20151345), the China Postdoctoral Science Foundation (Grant No. 2016M600381, 2016M601741), the Postdoctoral Science Foundation of Jiangsu Province (Grant No. 1601038C, 1601130B), the College Science
Foundation of Jiangsu Province (Grant No. 16KJB210003), the Priority Academic Program Development of Jiangsu Higher Education Institutions (PAPD), the Graduate practical innovation Foundation of Jiangsu province (Grant No. SJZZ16_0193), and the Undergraduate Scientific research Foundation of Jiangsu University (Grant No. 15A137). We would also like to thank many of our colleagues for many stimulating discussions in this field.

\section{References}

1 J. Yu, Z. Xu and T. Tan, Fuel Process. Technol., 2008, 89, 10561059.

2 J. M. Sablayrolles, A. Pandey, L. V. Rao and C. R. Soccol, Food Res. Int., 2009, 42, 418-424.

3 J. B. Doran, J. Cripe, M. Sutton and B. Foster, Appl. Biochem. Biotechnol., 2000, 84-86, 141-152.

4 Y. J. Wu, Y. Jin, Y. R. Li, D. Sun, X. S. Liu and Y. Chen, Vib. Spectrosc., 2012, 58, 109-118.

5 Q. S. Chen, J. R. Cai, X. M. Wan and J. W. Zhao, LWT-Food Sci. Technol., 2011, 44, 2053-2058.

6 H. Jiang, G. H. Liu, C. L. Mei and Q. S. Chen, Anal. Methods, 2013, 5, 1872-1880.

7 E. D. Louw and K. I. Theron, Postharvest Biol. Technol., 2010, 58, 176-184.

8 M. Blanco, A. C. Peinado and J. Mas, Biotechnol. Bioeng., 2004, 88, 536-542.

9 B. Finn, L. M. Harvey and B. Mcneil, Yeast, 2006, 23, 507-517.

10 A. X. Yang, J. L. Ding, H. L. Yan and K. Deng, Spectra Anal., 2016, 36, 691-696.

11 M. H. M. Killner, J. J. R. Rohwedder and C. Pasquini, Fuel, 2011, 90, 3268-3273.

12 K. Kim, J. M. Lee and I. B. Lee, Chemom. Intell. Lab. Syst., 2005, 79, 22-30.

13 S. K. Feng and H. J. Xu, Infrared Technol., 2008, 30, 58-60.

14 X. D. Sun, X. L. Dong, L. J. Cai, Y. Hao, A. G. Ouyang and Y. D. Liu, Sens. Lett., 2012, 10, 506-510.

15 G. B. Huang, H. M. Zhou, X. J. Ding and R. Zhang, IEEE Trans. Syst. Man Cybern. Part B Cybern., 2012, 42, 513-529.

16 A. Yeşilova, M. S. Özgökçe, R. Atlıhan, Ş. Polat Yıldız, I. Karaca and G. Ser, Fresenius Environ. Bull., 2016, 25, 1768-1778.

17 S. Calinon, F. D'halluin, E. Sauser and A. Billard, IEEE Robotics \& Automation Magazine, 2010, 17, 44-54.

18 J. W. Choi, S. H. Lee and S. G. Chung, Afr. J. Microbiol. Res., 2012, 6, 4620-4622.

19 M. C. A. Marcelo, C. A. Martins, D. Pozebon and M. F. Ferrão, Anal. Methods, 2014, 6, 7621-7627.

20 H. L. Zhang and Y. He, Spectra Anal., 2016, 36, 91-95.

21 W. Fan, Y. Shan, G. Y. Li, H. Y. Lv, H. D. Li and Y. Z. Liang, Food Anal. Method, 2012, 5, 585-590.

22 C. Xie, X. Ning, Y. Shao and Y. He, Spectrochim. Acta, Part A, 2015, 149, 971-977.

23 H. Li, Y. Liang, Q. Xu and D. Cao, Anal. Chim. Acta, 2009, 648, 77-84.

24 X. F. Yuan, Z. Q. Ge and Z. H. Song, Chemom. Intell. Lab. Syst., 2014, 138, 97-109. 
25 N. Abramson, D. Braverman and G. Sebestyen, IEEE Trans. Inf. Theory, 1963, 9, 257-261.

26 S. Calinon, F. Guenter and A. Billard, IEEE Trans. Syst. Man Cybern. Part B Cybern., 2007, 37, 286-298.

27 J. Q. Shi, R. Murray-Smith and D. M. Titterington, Int. J. Adapt. Control Sig. Process., 2012, 17, 149-161.
28 B. Muthén and K. Shedden, Biometrics, 1999, 55, 463469.

29 C. L. Mei, Y. Su, G. H. Liu, Y. H. Ding and Z. L. Liao, Chin. J. Chem. Eng., 2017, 25, 116-122. 